\title{
Implantologie
}

\section{Schonend und gestochen scharf}

Morita stellt dem Behandler mit dem digitalen Volumentomografen 3D Accuitomo 170 und dem Kombinationssystem Veraviewepocs 3D R100 2 High-End-Geräte zur Verfügung, welche eine sichere, schnelle und gleichzeitig gestochen scharfe Diagnostik gewährleisten. Alle bildgebenden Verfahren des Unternehmens sind so konzipiert, dass die Strahlenexposition nach dem sog. „ALARA-Prinzip“ (As Low As Reasonably Achievable) so gering wie möglich ausfällt. Diese Vorgabe steht stets im Fokus und manifestiert sich in innovativen Lösungen, welche die Dosis kontinuierlich weiter reduzieren - wie beim hochauflösende Kombinationssystem Veraviewepocs 3D R100, das sich für Panorama-, Cephalometrie- und 3D-Aufnahmen eignet. Sein R100-Sichtfeld ist als Reuleaux-Dreieck konzipiert und somit der natürlichen Form des Kieferbogens nachempfunden. Zur dentalen Weltleitmesse IDS 2015 hat Morita das Veraviewepocs 3D R100 zudem um 2 weitere Fields of View (FOV) erweitert. Eine sinnvolle Alternative zur Pla-

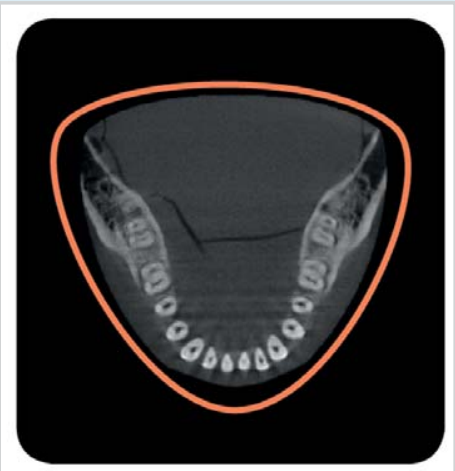
nung von implanto-

logischen und oralchirurgischen Behandlungen stellt der DVT 3D Accuitomo 170 dar, der auf die hochauflösende Darstellung komplexer Strukturen in allen 3 Raumebenen spezialisiert ist. Mittels insgesamt 9 zylindrischer Aufnahmevolumina mit Durchmessern zwischen $40 \mathrm{~mm}$ und $170 \mathrm{~mm}$ lassen sich alle Indikationsbereiche in Auflösungsabschnitten bis zu $80 \mu \mathrm{m}$ Voxel darstellen, detailliert dank der 4 unterschiedlichen Aufnahme-Modi wie dem Hochgeschwindigkeitsmodus „Hi-Speed“, der eine $180^{\circ}$-Aufnahme in nur 5,4 s erstellen kann. Weitere Infos zu den Hard- und Softwarelösungen von Morita unter www.morita.com/europe.

Nach einer Pressemitteilung der

J. Morita Europe GmbH, Dietzenbach 